\title{
Effect of motivative exercise based on the imaging of fNIRS and fMRI
}

\author{
Toshiyuki Tanaka1 \\ ${ }^{1}$ Department of Applied Physics and Physico-Informatics, Faculty of Science and Technology, Keio University, Japan \\ (Tel: +81-(0)45-566-1603; E-mail: tanaka@appi.keio.ac.jp)
}

Key words. Motivative Exercise, fNIRS, fMRI, Analytical Method, Rehabilitation

\begin{abstract}
In recent years, the stroke patients have increased in step with the aging of the population in many countries. Although "Takizawa method" was proposed as the new rehabilitation system for assisting physical therapists (PTs), the assessment is not sufficiently performed. The fNIRS and fMRI are useful for the assessment of rehabilitation effectiveness. Here, we discuss about assessment approach by using $\mathrm{NIRS}$ and $\mathrm{fMRI}$.
\end{abstract}

\section{Background}

When the activating site in brain is clearly measured, an imaging by $\mathrm{AMRI}$ is good selection. However, fMRI have the following restrictions; do not place the metallic device near the fMRI and do not move under inspection. Although those restrictions are disadvantageous for the assessment under rehabilitation, ANIRS [1, 2] is better for using in the rehabilitation. Since the imaging of fNIRS is mapped only on the cephalic surface, the activating site in brain must be obtained by computer analysis. In this research, we propose the analysis method of activating site in brain and effect verification method for motivative exercise.

\section{Method}

The activation sites and failed part in brain of stroke patients are first measured by fMRI, as shown in Fig. 1 (but, the figure is obtained from the healthy person). We perform the Takizawa method for stimulating the failed region by easy devices. Since the fMRI is not used in the rehabilitation with devices, ANIRS is used for measuring in the recovery level. The imaging by fNIRS is shown in Figs. 2 to 4; Fig. 2 is the position of detector of fNIRS, Fig. 3 shows the result of signal processing by Bonferroni's method, and Fig. 4 shows the mapped imaging on cephalic surface. The activation site in brain is estimated from the data of $\mathrm{fNIRS}[3,4]$ as shown in Fig. 5. We can evaluate the brain function and recovery level by comparing the estimated activation site and the failed region obtained by fMRI.

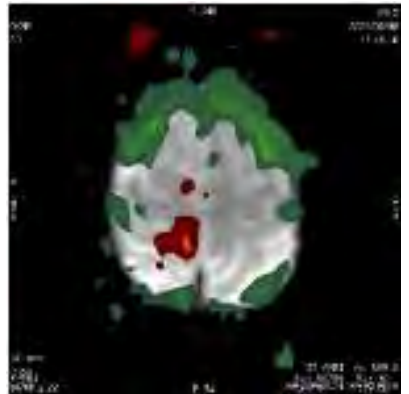

Fig. 1 fMRI image

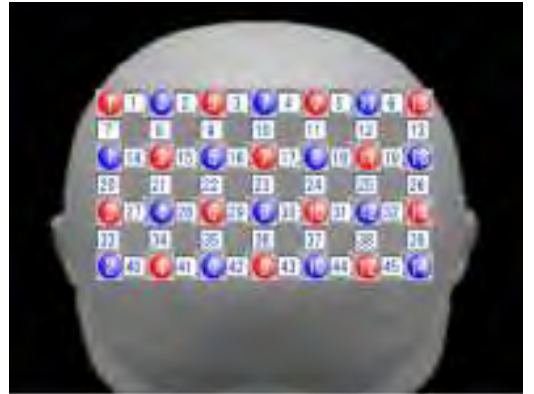

Fig. 2 Detector position on head

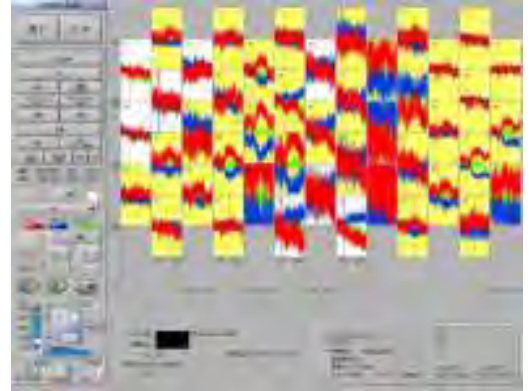

Fig. 3 Bonferroni's Method

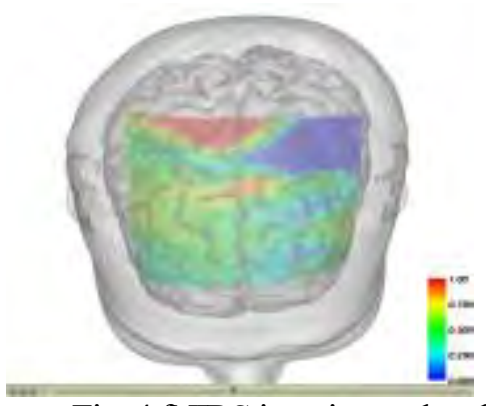

Fig. 4 fNIRS imaging on head

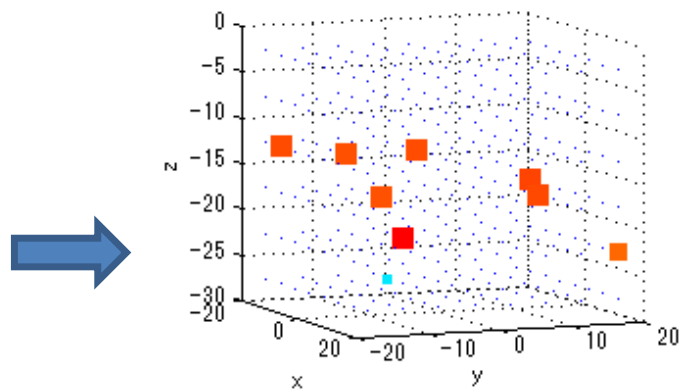

Fig. 5 Estimation of activating site

\section{Discussion}

We have tried to obtain the position of activation site in the deep part of brain by using the mathematical method of inverse problem. As the matter of course, we will carry on the approach of measuring the precise activation site. Here, we consider the future research for Takizawa method rehabilitation. We show the following trial for rehabilitation in Tanaka laboratory.

(1) Brain function measurement of stroke patient by $\mathrm{ANIRS}$ in the rehabilitation and AMRI 
(2) Discriminant analysis of Takizawa method experimental data

\section{Reference}

[1] Priori Alberto, Bertarelli Alfredo, Rona Sabine, Accornero Neri, Manfredi Mario, "Polarization of the human motor cortex through the scalp", Neuroreport, Vol. 9, pp. 2257-2260, 1998

[2] Nitsche MA, Paulus W, "Excitability changes induced in the human motor cortex by weak transcranial direct current stimulation", The Journal of Physiology, Vol. 572, pp. 633-639, 2000

[3] David A. Boas and Anders M. Dale, "Simulation study of magnetic resonance imaging-guided cortically constrained diffuse optical tomography of human brain function”, Applied Optics, Vol. 44, Issue 10, pp. 1957-1968, 2005

[4] Eiji Okada, Michael Firbank, and David T Delpy, "The effect of overlying tissue on the spatial sensitivity profile of nearinfrared spectroscopy”, Physics in Medicine and Biology, Vol. 40, No. 12, 1995 